\title{
La gestión del Marketing \\ que conecta con \\ los sentidos
}

FECHA DE RECEPCIÓN: 9 de agosto FECHA DE APROBACIÓN: 25 de octubre Pp. 168-183

Claudia Gómez R.* Jorge Eduardo Mejía*

\section{The market management which connects with the senses}

La gestion mercatique qui connecte

les sens

* Ingeniera Industrial de la Pontifica Universidad Faveriana, Magister en Gestión de Organizaciones de la Universidad de Québec a Chicoutimi y de la Universidad EAN;

A gestãu du marketing Especialista en Mercados de la que liga $\mathrm{com}$ os sentidos

** Diseñador Industrial de la Universidad Autónoma de Manizales (1995) y Magister en Administración del Instituto Tecnológico y de Estudios Superiores de Monterrey - México (2003). Profesor Investigador Colegio de Estudios Superiores de Administración - CESA. Miembro del Grupo de Investigación en Innovación y Gestión Empresarial (GIIGE) en la Línea de Investigación en Mercadeo. 


\section{RESUMEN}

El poder de los sentidos sobre percepciones y emociones de los individuos, ha sido tema de estudio dada la incidencia que ejercen sobre el comportamiento de compra y consumo de productos y servicios. De allí nació la aplicación de lo sensorial en el Marketing, la cual exige una adecuada gestión para sacar el provecho de los beneficios otorgados a la respuesta de la estimulación sensorial, que finalmente se traducirán en ventas rentables para la compañia. Así el objetivo general propuesto en esta investigación consistió en identificar y comprender los factores a tener en cuenta en una gestión de marketing sensorial, partiendo de diferentes propuestas usando los sentidos que se han desarrollado a la fecha en algunas partes del mundo y en Colombia. Como objetivos específicos se pretendió construir una línea de tiempo del estudio sobre los sentidos, identificar la relación de los sentidos con el proceso de compra, los objetivos y estrategias perseguidos con el marketing sensorial y determinar un modelo usado en su aplicación.

\section{ABSTRACT}

The power of the senses in terms of perceptions and emotions of individuals has been a research theme due to the incidence exerted upon buying and consuming behavior of products and services. From there, the application of a sensorial perspective to marketing was born, which demands from adequate management, which takes advantage given as an answer to sensorial stimulation and which finally shows profitable sales for the company. The main objective of this research aims at identifying and interpreting the factors to bear in mind for sensorial marketing management, starting with some proposals on the use of senses that have been currently developed in some parts of the world and in the country of Colombia. As specific aims, we can mention: to design a time- line in the study of the senses, to identify the relationship of the senses in the buying act, the stated aims and strategies for sensorial marketing, and to select a model to be used in its application.

\section{RESUMÉÉ}

Le pouvoir des sens sur les perceptions et les émotions des individus a été sujet de nombreuses études compte tenu de son impact sur le comportement d'achat et la consommation de produits et services. De là est né l'utilisation du sensoriel en mercatique qui nécessite une gestion adéquate permettant de tirer profit des avantages accordés à la réponse de stimulation sensorielle, qui se traduit par des ventes rentables pour l'entreprise. Ainsi, l'objectif général de cette étude est d'identifier et de comprendre les facteurs à prendre en considération pour une gestion efficace de la mercatique sensorielle. Cette gestion se fait sur la base de différentes propositions utilisant les sens élaborés à ce jour dans certaines parties du monde et en Colombie. Les objectifs spécifiques visent à établir une chronologie de l'étude sur les sens, identifier la relation sensorielle sur le processus d'achat, les objectifs et stratégies mercatiques sensorielles afin de déterminer un modèle utilisable par l'application.

\section{RESUMO}

O poder dos sentidos sobre percepções e emoções dos indivíduos tem sido tema de estudo por sua incidência sobre o comportamento de compra e consumo de produtos e serviços. Daqui nasceu a aplicação do sensorial no marketing, que exige uma adequada gestão para aproveitar os benefícios dados à resposta de estimulação sensorial, que finalmente levarão a vendas rentáveis para a empresa. O objetivo geral proposto nesta pesquisa consistiu em identificar e compreender os factores a considerar numa gestão de marketing sensorial, partindo de diferentes propostas usando os sentidos, que tem sido desenvolvidas até $\grave{a}$ data em alguns lugares do mundo e na Colômbia. Como objetivos específicos pretendeu-se construir uma linha de tempo do estudo sobre os sentidos, identificar a relação dos sentidos com o processo de compra, os objetivos e estratégias procurados com o marketing sensorial e determinar um modelo usado para sua aplicação.

\section{Palabras claves}

Marca

Marketing sensorial

Gestión

Multisensorial

Sentidos

\section{Key words}

\author{
Branding \\ Sensorial marketing \\ Management \\ Multisensorial \\ Senses
}

\section{Mots clefs}

\author{
Marque \\ Mercatique sensorielle \\ Gestion \\ Multisensoriel \\ Sens
}

\section{Palavras-chave}

\author{
Marca \\ Marketing sensorial \\ Gestão \\ Multisensorial \\ Sentidos
}




\section{INTRODUCción}

E n una gestión estratégica ha de tenerse en cuenta lo que sucede en el entorno, con miras a dar respuesta pertinente y coherente entre las capacidades de la compañía y su razón de ser (Hitt, Black, y Porter, 2006). Una respuesta pertinente es aquella que permite aprovechar las oportunidades y preparase para enfrentar amenazas.

El entorno de negocios que enfrentan actualmente los gerentes de Marketing presenta cambios acelerados tales como incremento de la competencia por la atención del consumidor, mayor innovación que impide la perdurabilidad de las ofertas entregadas, velocidad de las comunicaciones que ya permiten ser en tiempo real y, unos consumidores cada vez más educados en las diferentes estrategias y tácticas practicadas por los gerentes de Marketing.

Al enfrentar este escenario y explorar alternativas de respuesta a estas exigencias, los gerentes de Marketing han encontrado que la importancia relativa de los sentidos frente a las marcas específicamente en la exposición de cada uno de ellos se comporta así: el sentido de la vista ocupa el $58 \%$ el olfato el $45 \%$, el oído el $41 \%$, el gusto $31 \%$ y el tacto $25 \%$ (Álvarez del Blanco, 2011). De ahí que muchas de las marcas quieran explorar cada día más los sentidos del olfato y del oído, pues amplían las esferas de percepción de los consumidores tanto de los espacios y ambientes comerciales como de las relaciones de identidad con las marcas, y el valor del producto (Morrin, 2010 en Krishma, 2010).

Pero además de la percepción, el uso del estímulo de los sentidos influye sobre las emociones y la memoria del ser, lo que permite lograr en el largo plazo relaciones afectivas y mayor permanencia de la marca. Estas experiencias gerenciales como nuevas maneras de presentar e influenciar la acción de compra, han mostrado un gran impacto que crece cada día más. De acuerdo con Álvarez del Blanco (2011), se estima que el $40 \%$ de las empresas que aparecen en el Ranking de Fortune 500 implementarán algún tipo de estrategia de Marketing sensorial en los próximos años.

Es así como en un entorno cada vez más competitivo, las empresas que quieran generar diferenciación en el largo plazo deben comenzar a pensar en su marca, su producto, sus empaques y sus espacios no sólo desde la información y la comunicación visual, sino desde la generación de sensaciones que estimulen cada uno de los sentidos y que además inviten a los consumidores a identificarse con los aromas, sonidos y ambientes de los productos y servicios. 


\section{METODOLOGÍA}

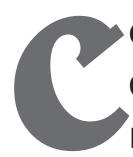

mo metodología se realizó una investigación de tipo documental y exploratoria mediante revisión de material impreso publicado o que reposa en bibliotecas particulares. Específicamente se utilizaron como base, textos, videos, páginas Web sobre el tema sensorial y material de investigación -incluido trabajo de campo-, desarrollado por los investigadores en el CESA. Se procedió a triangular la información encontrada en las diversas fuentes para llegar a una propuesta concreta sobre factores y estrategias del

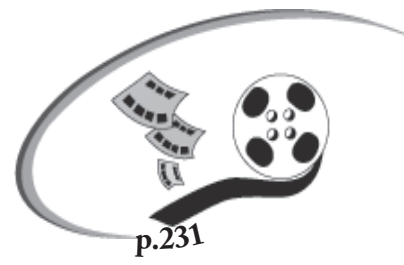
Marketing sensorial que deben tenerse en cuenta en la gestión.

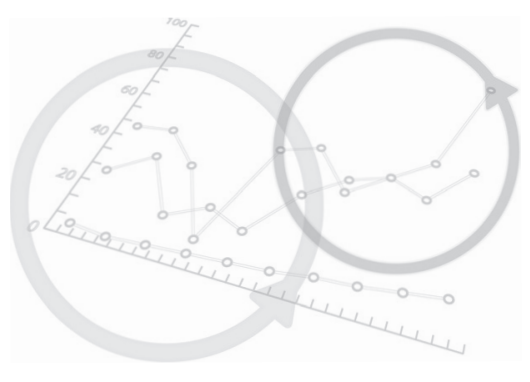

\section{RESULTADOS}

\subsection{Evolución histórica de estudios de los sentidos}

Al construir una crónica de estudios de los sentidos basada en registros de las fuentes consultadas, se encontró que se inicia en 1909 con la identificación de la existencia de relaciones entre sentidos, como es la creación de imágenes a partir de una percepción olfativa. En 1947, se encontraron estudios sobre los procesos mentales tales como percepción, sensaciones, asociaciones mentales, memoria y en general acciones e interacciones entre los sentidos. Pero es a partir de este milenio, que se registran estudios sobre temas relacionados

con el enganche emocional a través de los sentidos y el uso del Neuromarketing tales como los registrados a continuación:

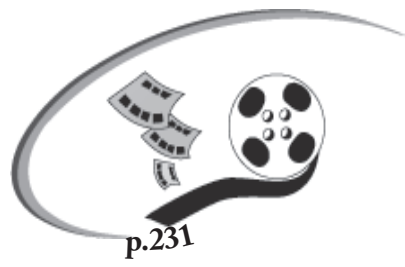

- Branding emocional (Gobé, 2001).

- El odotipo y el Marketing olfativo (Bonadeo, 2005).

- Los sentidos y la cautivación emocional en la compra (Lindstrom 2005 y 2009). 
- Neuromarketing y motivos de compra (Braidot, 2008).

- Marketing sensorial (Hultén, et alt, 2009).

- Marketing experiencial (Schmitt, 2006).

- Las reacciones de los consumidores y los sentidos (Elder et alt, 2010).

- Marketing sensorial (Krishma, 2010).
- La mente subconsciente como el cerebro que compra (Pradeep, 2010).

- Estrategias de Branding hoy y el Neuromarketing (Álvarez del Blanco, 2011).

- Tendencias del Marketing sensorial (Resa, 2011).

A continuación, se puede apreciar una línea del tiempo sobre estudios de los sentidos en procesos mentales y su aplicación en los actos de compra y consumo de productos y servicios (figura 1).

Figura 1. Línea del tiempo marketing sensorial

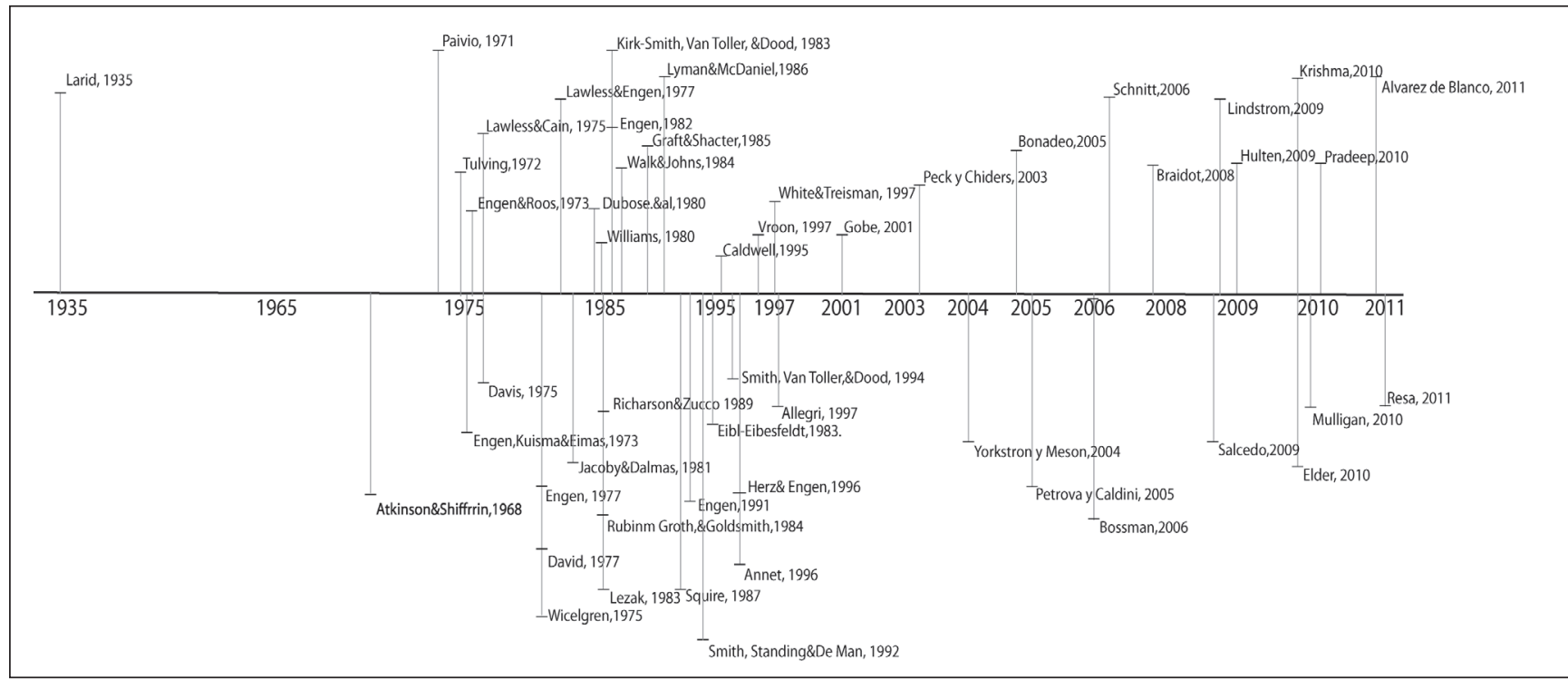

Fuente. Elaboración propia (2012).

\subsection{Los sentidos y la gestión del proceso de compra}

La sensación se refiere a experiencias inmediatas básicas, generadas por estímulos aislados simples y también se define en términos de la respuesta de los órganos sensoriales frente a un estímulo. Tradicionalmente han sido vinculadas a los cinco sentidos definidos por Aristóteles: vista, oído, olfato, gusto y tacto (Álvarez del Blanco, 2011b).

El sentido del olfato: no es filtrado por el proceso racional de un individuo (Bonadeo, 2005 y Gavilán, 2011) y permite la generación de vivencias plenas de sensaciones como respuesta a las percepciones estimuladas por el aroma. Así que, estas son la base para las relaciones de marca asociadas a las diversas categorías de productos ligados a diversos aromas que producen reacciones en los individuos, buscando que el consumidor relacione la marca con determinado aroma, tanto previo a la compra del producto como durante la compra y el consumo del mismo. Su potencial resulta del hecho mediante el cual se puede tener una imagen visual de un aroma, tal como lo expresó Gamble: "a pesar de 
que puede ser difícil tener una imagen mental de un aroma, muchos escritores afirman que sólo basta con oler una fragancia determinada para revivir un recuerdo complejo y lleno de emociones" (Lawless, 1975 en Bonadeo, 2005).

El sentido del oído: la música, relacionada directamente con los estados de ánimo y la generación de recuerdos a largo plazo, puede producir emociones, sentimientos y experiencias en las personas y hacer que estas actúen de manera diferente en diversos ambientes dependiendo del tipo de música que esté sonando en un momento dado. También es de resaltar que el sonido influye sobre la percepción del sabor y de la frescura del alimento, según Zampini y Spence (en Avello, Gavilán y Abril, 2011). Igualmente, el oído se puede asociar con otros sentidos, al punto de provocar sinestesias, esto es, oír colores, olores o sabores (Avello, Gavilán y Abril, 2011).

El sentido de la vista: es uno de los sentidos que más puede impactar nuestra mente, pues facilita la persuasión y genera gran capacidad de recordación. Es el sentido más estimulado desde la perspectiva del Marketing, con aplicaciones en empaques, avisos comerciales, diseño de puntos de venta y páginas Web. En este sentido, se combinan los factores emocionales y racionales que alteran la toma de decisiones de compra (Costa, 2010).

El sentido del gusto: es el sentido menos explorado desde el Marketing sensorial, y está limitado alámbito de la gastronomía en la selección de comidas y bebidas, las catas de vinos, cafés principalmente. Es un sentido muy personalizado en el que parte del objeto de análisis debe entrar en contacto con las papilas especializadas de la lengua; trabaja conjuntamente con el olfato, pues entre el 80 y el $90 \%$ del sabor procede del olor (Gavilán et al, 2011).
4 El sentido del tacto: los materiales y las texturas se perciben a través del sentido del tacto en donde los consumidores pueden verificar lo que están comprando, pues les permite tener una primera percepción de la calidad del producto. La información relacionada con la textura, la dureza, la temperatura y el peso, está relacionada con as-pectos fundamentales en algunas categorías de productos; por ejemplo, sentir el peso del teléfono celular proporciona un indicador de calidad que no se puede tener por medio de sólo imágenes o compras por Internet, al igual que sentir la textura de los tomates en el supermercado o el material de una chaqueta de cuero. Estos aspectos ayudan al consumidor a percibir relaciones de calidad, integrando de manera más eficaz la información reforzada por los otros sentidos, además que al actuar con alguno de los otros sentidos -por ejemplo la vista para complementarse-, que por lo general es así, genera experiencias multisensoriales (Serra, Manzano y Avello, 2011).

Además de los sentidos, enriquece saber que hay estudios que demuestran que el acto de decisión de compra dura 2.5 segundos (Álvarez del Blanco, 2011). De modo que se cuenta con muy poco tiempo para impactar y destacarse ante los sentidos del consumidor; y esto es lo que se traduce en una necesidad imperiosa tanto identificar el o los componentes sensoriales de la marca, como el desarrollar la representación de la marca por el canal sensorial o canales sensoriales más adecuados como factor de atracción.

\subsection{La gestión: objetivos y estrategias}

Los responsables de Marketing en su gestión deben ser capaces de diseñar y administrar los procesos implicados

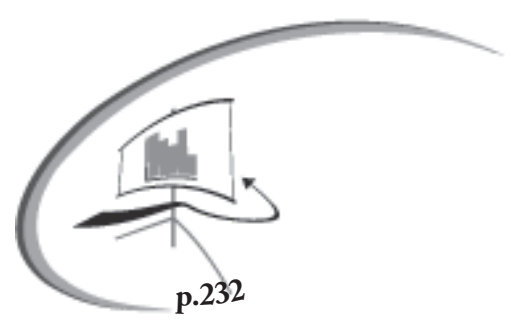


en las actividades de Marketing, a través de sus tres funciones básicas, a saber: "comprender consumidores, conquistar compradores y conservar clientes" (Asociación Colombiana de Facultades de Administración -Ascolfa e Instituto Colombiano para el Fomento de la Educación Superior-Icfes, 2010). Así que para la comprensión, la conquista y la conservación debe tener dominio sobre los factores que afectan las decisiones de compra de los compradores y las de consumo de los consumidores y, en lo que a Marketing sensorial se refiere, le exige total conocimiento del funcionamiento de cada uno de los sentidos (vista, oído, olfato, gusto y tacto) y la reacción del consumidor a su estimulación externa. Para lograrlo se apoya en Neuromarketing, que aporta al conocimiento de las reacciones emocionales del ser humano (Lindstrom, 2009).

Esa comprensión del comportamiento emocional del consumidor le permite al gerente de Marketing establecer un diagnóstico que será la base para su planeación estratégica. En esta, los objetivos como generar recordación, compromisos en el largo plazo, o una alta diferenciación se mantienen con respecto a la gestión tradicional, pero las estrategias para lograrlos incluyen el uso de la tecnología como el neuromarketing y la innovación como el uso de los sentidos en manejo de los aspectos emocionales en las decisiones de compra de los consumidores, en la creación de nuevas sensaciones o énfasis de alguna de las ya existentes, lo que puede incrementar el atractivo del producto o servicio, en donde está implícita la marca. Se considera innovadora puesto que fue en el presente milenio cuando las empresas han estado enfocando esfuerzos al desarrollo de los aspectos sensoriales de los productos buscando el enganche emocional con el consumidor (Krishna, 2010).

El uso de estimulación de los sentidos se fundamenta en el hallazgo de que las sensaciones y las decisiones irracionales priman sobre la estructura tradicional (necesidad-beneficio) (Pradeep, 2010). Esa perspectiva sensorial, incluye la estimulación de uno o varios de los cinco sentidos (vista, oído, tacto, gusto y olfato), mediante los cuales se pretende llegar al consumidor por medio de experiencias sensoriales que permitan influir en su acción de compra y establecer relaciones de largo plazo entre él y la marca (Gobé, 2001; Hultén, Broweusss y Van Dijk, 2009).

Estas estrategias de estimulación múltiple de los sentidos pueden contemplarse en el diseño del producto, de su empaque, de su marca, del ambiente en que puede comprar y en el que se puede consumir y/o de la comunicación. Se trabaja sobre la estimulación de los sentidos con miras a manejar las percepciones, puesto que por ejemplo variables ligadas a la calidad y la funcionalidad en el diseño de producto ya no son suficientes para que el consumidor tome decisiones de compra (Braidot, 2008). Tampoco son suficientes las ya tradicionales estrategias empleadas dentro del marketing ligadas a los medios impresos, la TV, la radio y la publicidad exterior desde un enfoque racional, el uso de los sentidos como canales de comunicación, se está convirtiendo cada día más en parte de la gestión que complementa.

Así que para dar respuesta al nuevo consumidor se requiere la generación de vínculos emocionales, que se convierte en un componente único e irrenunciable en las decisiones de compra, y es, en este punto, donde la gestión a través de las diversas estrategias de marketing debe tener claridad en cuales de los aspectos del Marketing sensorial se debe profundizar. Y no solo sobre cuál aspecto enfatizar, sino que es necesario tener en cuenta también todo el ciclo de la compra, en donde el consumidor vive una experiencia: en la pre-compra, otra en la compra y otra en la pos compra.

\subsubsection{Objetivos del Marketing sensorial}

Puede analizarse el momento del proceso o ciclo de la compra, lo que permite identificar alternativas de objetivos que se pueden perseguir usando lo sensorial en el Marketing, por ejemplo en: 
- La pre-compra puede trabajarse buscando desarrollar notoriedad o expectativas y/o potenciar la relevancia y captura de atención de las personas, optimizar la comunicación de asociaciones de marca.

- La compra específicamente, persigue los objetivos de maximizar la percepción de valor, la preferencia y lograr la sorpresa y deleite de la compra.

- La post-compra, tiene como objetivo, maximizar la experiencia de consumo o uso y fidelizar al consumidor (Manzano, marzo/abril 2011).

La coherencia a través de todo el proceso permite conseguir los objetivos de construir experiencias duraderas en el largo plazo, de lograr el enganche emocional a través de los sentidos, y facilitar la toma de decisiones de los consumidores mediante la generación de recordación o una alta diferenciación.

\subsubsection{Estrategias de Marketing sensorial}

Parte del proceso en la construcción y adecuada gestión de una marca radica en definir las diversas estrategias de Marketing que sean congruentes con lo que ella quiere proyectar. En cuanto a estrategias del Marketing sensorial, se encuentran específicamente:

- La conexión con la imagen que genera identidad, en las diferentes actividades de comercialización. El Branding sensorial para construir la imagen de marca a través del desarrollo de experiencias sensoriales implicando a varios de los cinco sentidos.

- Uso concentrado o una mezcla de los sentidos, que se pueden utilizar para la marca. En este orden de ideas es necesario determinar una prioridad de los sentidos que se consideraron posibles a explotar, lograr sinergias y efectos cascada.
En la estrategia de mezcla de sentidos, se genera una marca multisensorial, entendida como aquella que explota las percepciones de todos los sentidos humanos. Pero para ser exitosa debe haber congruencia entre los diferentes estímulos y la esencia de la marca. Se destaca que cuando hay mayor percepción sensorial, se ha encontrado como resultado mayor recordación y fidelidad:

una serie de estudios demuestran que la fidelidad promedio de la marca se incrementa un $28 \%$ cuando uno de los sentidos se activa positivamente y hasta un $43 \%$ cuando la marca se apoya en 2 ó 3 aspectos sensoriales, y alcanza $58 \% 4$ o 5 sentidos (Álvarez del Blanco, 2011a).

Esta decisión de gestión al respecto de desarrollar la marca a un nivel multisensorial, estará en función de las experiencias que incrementan valor a los productos desde el punto de vista de los consumidores.

- Establecimiento de una firma sensorial, lo que implica un desarrollo específico para el reconocimiento y diferenciación de un producto o marca tanto desde lo visual (logosímbolo), como lo sonoro (fonotipo), lo olfativo (odotipo), lo gustativo (sabor) o lo táctil.

- Definición de la intensidad de cada una de las variables multisensoriales cuando se ha decidido trabajar una mezcla de las mismas (Álvarez del Blanco, 2011).

\subsubsection{Gestión de los estímulos sensoriales}

En todos los casos independientemente del canal que se use, se está trabajando la comunicación con el consumidor y el estímulo que se trabaje ha de tener el suficiente atractivo para que sea captado por el consumidor, que en este caso es el interlocutor. Tampoco debe ser tan exagerado que impida la aplicación correcta de los demás con los demás 
sentidos, como en el caso particular del vino (Peris y Cuadrado, 2008). Igualmente ha de trabajarse como algo habitual y consistente, para que tenga el efecto de largo plazo.

El uso de la estimulación por los sentidos debe trabajar alineado con la identidad de marca perseguida, de modo que fortifiquen la marca y generen diferenciación y sorpresa en el consumidor para deleitar la experiencia no sólo de compra, sino la del consumo o uso del producto o servicio.

\subsection{Modelo del Marketing sensorial}

Desde lo operacional, se puede afirmar que el modelo cuenta con cuatro pilares básicos: relaciones sensoriales, experiencias sensoriales, imaginación sensorial y visión de marca.

Las relaciones sensoriales están ligadas a los diálogos emocionales que implican sensaciones, emociones, percepciones y estímulos y el contacto con los usuarios. Es a partir de la relación entre la cognición y las emociones que se identifican las diferentes maneras en que los consumidores se aproximan a los productos teniendo estos, un conjunto de deseos y emociones que tienen gran influencia en la selección de los objetos y crean preferencias basadas en la percepción sensorial. Esto lleva a establecer relaciones basadas en categorías de color, forma, textura, marca (Eysenck, 1992).

Las experiencias sensoriales responden a la estimulación de los sentidos tanto en la compra como en el consumo de forma congruente en todas las manifestaciones de la marca: producto, punto de venta, servicio. La imaginación sensorial genera la sorpresa y deleite del consumidor. Así que los procesos de Marketing sensorial a partir de la estimulación de múltiples sentidos se basan en desarrollar permanentemente la imaginación, de tal forma que puedan integrarse tanto los medios tradicionales, como en las nuevas tecnologías a través del internet, las redes sociales, las aplicaciones en teléfonos inteligentes, entre otros. Todo en la búsqueda de la activación de emociones, sentimientos, sonrisas, y generar empatía e identidad con las marcas por medio de la imaginación, mucho más allá del espacio comercial.

Finalmente, el pilar de la visión de marca está relacionado con el largo plazo tanto en orientación como en evolución. Entender la visión de marca desde el Marketing sensorial permite visualizar a los clientes y no clientes pensando en establecer conexiones emocionales con todas las personas así no puedan acceder al producto o servicio en el corto plazo. Las marcas que inspiran, que nos hacen reír, aquellas marcas con las que se sueña usualmente han mantenido una estrategia de largo plazo ganando reconocimiento y posicionamiento no solo en nuestras mentes sino también en nuestros corazones; un ejemplo de esto lo podemos encontrar en marcas como Apple, que se convierten en íconos que generan rituales, identidad y convierten la experiencia de compra en todo un suceso que no se olvida fácilmente.

\subsection{Casos aplicados}

Se analizaron diversas experiencias con el fin de identificar los diferentes

enfoques de gestión

detallando objetivos

perseguidos, estrategias

empleadas y los sentidos que más se están explorando al momento de la revisión del tema, en diversas categorías de productos y servicios. Se aprecian diferentes ejemplos, que

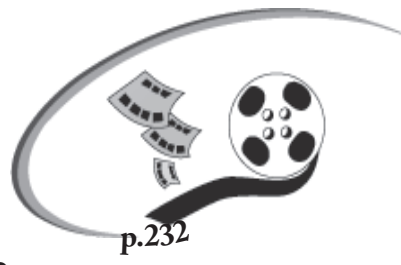
incluyen categorías de alimentos, bebidas, turismo, y tecnología (tabla1). 
Tabla 1. Experiencias aplicadas

\begin{tabular}{|c|c|c|c|}
\hline Marca & Estrategia sensorial & Sentidos estimulados & Objetivo perseguido \\
\hline Starbucks & Multisensorial. & $\begin{array}{l}\text { Auditivo } \rightarrow \text { música. } \\
\text { Olfativo } \rightarrow \text { aroma. } \\
\text { Visual } \rightarrow \text { colores de } \\
\text { empaques. } \\
\text { Táctil } \rightarrow \text { materiales de } \\
\text { empaques. } \\
\text { Gustativo } \rightarrow \text { sabores café. }\end{array}$ & $\begin{array}{l}\text { Hacer sentir al visitante como } \\
\text { en su casa, muy relajado, y } \\
\text { cautivarlo emocionalmente } \\
\text { (Hultén, Broweus, \& Van } \\
\text { Dijk, 2009). }\end{array}$ \\
\hline Bon Bon Bum & $\begin{array}{l}\text { Trisensorial: gusto, olfato, } \\
\text { visión. }\end{array}$ & $\begin{aligned} \text { Olfato } \rightarrow & \text { aromas. } \\
\text { Gusto } \rightarrow & \text { sabores y rellenos } \\
& \text { de sabores. } \\
\text { Visual } \rightarrow & \text { colores y tinturas. }\end{aligned}$ & $\begin{array}{l}\text { Identificación y preferencia } \\
\text { del grupo objetivo, (Mejía, } \\
\text { 2011a). }\end{array}$ \\
\hline $\begin{array}{l}\text { SONY - PS2la - 2005/ } \\
\text { Malasia }\end{array}$ & Un sentido concentrado (tacto). & $\begin{array}{l}\text { Táctil } \rightarrow \text { pantallas simuladas } \\
\text { con textura de plástico de } \\
\text { burbujas de aire -usados en } \\
\text { embalajes de productos deli- } \\
\text { cados y que gustan a la gente } \\
\text { para explotar con los dedos } \\
\text { (Aromarketing, 2011; } \\
\text { vidaextra.com, 2009). }\end{array}$ & $\begin{array}{l}\text { Incrementar confianza en la } \\
\text { marca y producto y por tanto } \\
\text { su valor percibido. }\end{array}$ \\
\hline $\begin{array}{l}\text { Singapore Airlines } \\
\text { aeronaves }\end{array}$ & Un sentido concentrado (olfato) & $\begin{array}{l}\text { olfato } \rightarrow \text { aroma como odotipo } \\
\text { de la marca- Stefan Floridian } \\
\text { Waters. (Aromarketing, 2011) }\end{array}$ & $\begin{array}{l}\text { Generar experiencias pla- } \\
\text { centeras y únicas tanto vivi- } \\
\text { das como recordadas. }\end{array}$ \\
\hline Chocorramo & $\begin{array}{l}\text { Un sentido concentrado } \\
\text { (visual). }\end{array}$ & $\begin{aligned} \text { Visual } \rightarrow & \text { colores y transpa- } \\
& \text { rencia del empaque. }\end{aligned}$ & $\begin{array}{l}\text { Percibir y visualizar el pro- } \\
\text { ducto como hecho en casa, } \\
\text { artesanal y no industriali- } \\
\text { zado, (Mejía, 2011b). }\end{array}$ \\
\hline
\end{tabular}

Fuente. Elaboración Propia (2012).

En estos ejemplos se vieron estrategias multisensoriales, estrategias de combinación de dos o tres sentidos y estrategias de concentración en un sentido (en algunos casos el olfato y en otro el tacto). Estas propuestas están relacionadas con la evocación de emociones del grupo objetivo, a la vez que están potenciando la experiencia de consumo o uso del momento con miras a fortalecer la recordación de las mismas, lo que incide sobre la percepción de valor de la marca.

Usando como base el modelo de Marketing sensorial de los cuatro pilares se procedió a aplicarlo a un caso nacional (el Bon Bon Bum) con miras a profundizar en el análisis del mismo, el caso del mismo.

- Imaginación sensorial. Trabajó la sorpresa mediante la inclusión de chile, sabores ácidos y colores que teñían la lengua. Inicialmente generó un nuevo concepto el de la chupeta esférica o de bola rellena de chicle. Es el bombón que hace Boom (Mejía, 2011a) y que originó el nombre de marca. Como dulce de larga duración, en comparación a las demás chupetas en su momento en el mercado, mientras se derrite da lugar a la imaginación y el juego por parte de sus consumidores. 
- Relaciones sensoriales. A través de los diálogos emocionales trabajó la sensación de lo duro a lo blando; emociones ligadas al juego a través del chicle (haciendo bombas más grandes o más pequeñas y que al reventar producen sonido de estallido); percepciones en la infancia de alegría surgida de piñatas y consumo de golosinas, mientras que en la adolescencia están asociadas a sensualidad y erotismo, y los estímulos sensoriales ligados a las papilas gustativas, trabajando sobre la vista. Se mantiene contacto intenso con los consumidores a través de la publicidad, que hace énfasis en la tradición familiar e igualmente relación con los cajoneros, trabajando con mayor énfasis la promoción de venta mediante obsequios.

- Experiencias sensoriales. En la experiencia de consumo, a través de la estimulación de los sentidos de la vista, el gusto y el olfato esperando el juego con el chicle y la sorpresa de los rellenos por parte de los infantes y el juego de la conquista por su forma para los adolescentes. En cuanto a la compra, se trabaja desde la exhibición en la precompra, el lenguaje del color del empaque en la compra y hasta lo ambiental en la post- compra.
- Visión de marca. Desde la misión de la compañía se hace mención al sabor de la vida y en la visión a los alimentos gratificantes (Mejía, 2011a). Siempre con la novedad, se conecta emocionalmente con sus consumidores a través de nuevas sensaciones y nuevo uso del producto por los sabores, colores, texturas visuales, partículas flotantes de frutas y tintes que añaden y cambian a cada nuevo producto de la línea. Así mismo, se vincula a la preservación del medio ambiente y genera los mensajes responsables ligados a la conservación del planeta como compromiso de la marca y por su puesto empresarial. El posicionamiento que ha logrado a través de los años y a través de los mensajes publicitarios ha sido el de golosina divertida, juguetona y juvenil, pues la marca se ha renovado mediante sus diferentes versiones acorde con tendencias y modas pero filtradas con el consumidor con lo que ha logrado su identificación con el mercado meta (Mejía, 2011a).

Al integrar estos pilares coherentes entre sí, se puede apreciar la respuesta que se da a los objetivos perseguidos de Identificación y preferencia del grupo objetivo.

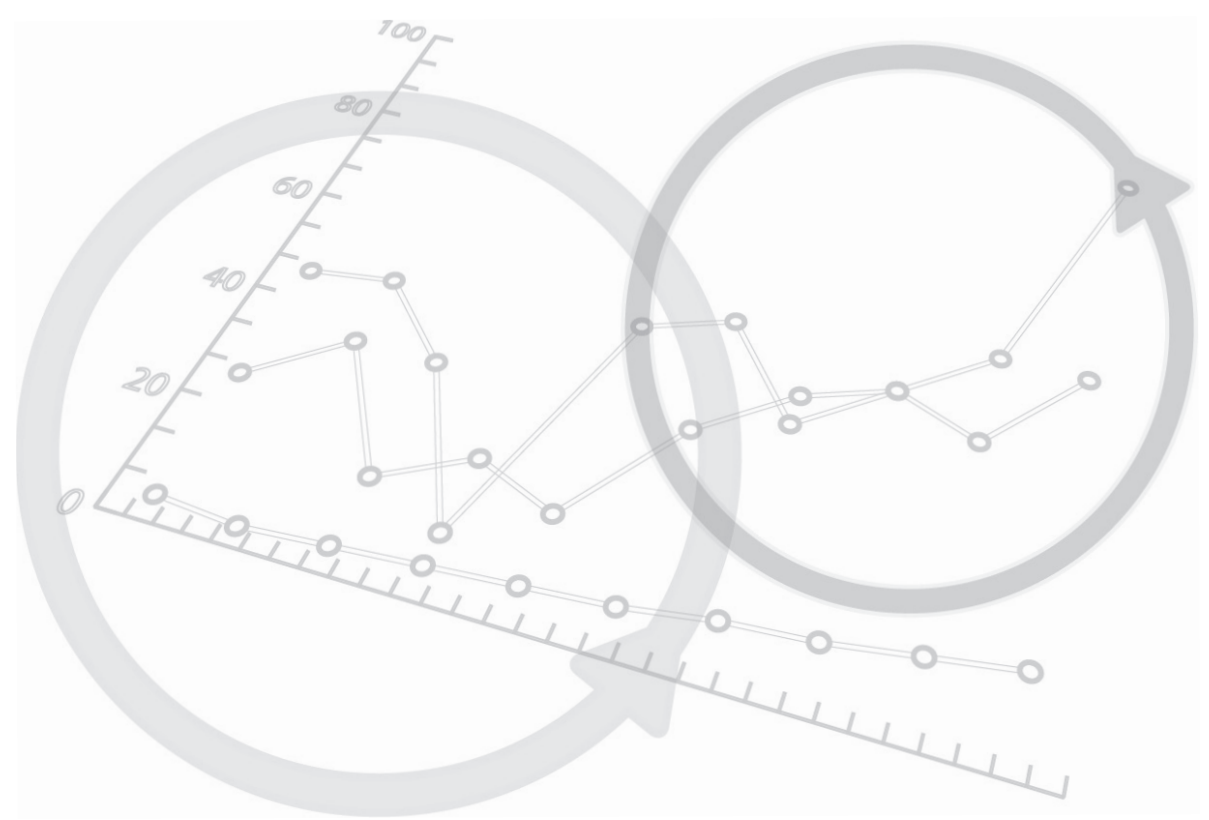




\section{CONCLUSIONES}

- El mercado y el entorno exigen cada vez mayor diferenciación y novedad. Esto lo que ha implicado en la investigación el desarrollo de nuevas herramientas para conseguir información que fundamente las decisiones en la gestión del marketing sensorial.

- En la gestión del marketing, el análisis del consumidor a profundidad es un requisito indispensable para identificar las relaciones asociaciones y percepciones que tienen estos con olores, sonidos y texturas marcados desde su cultura, formación y experiencias vitales.

- La gestión del Marketing sensorial exige conocer y comprender los aspectos emocionales que se generan con los estímulos sensoriales, para tener una base de diagnóstico desde lo sensorial y proceder con la planeación. Esto implica nuevos conocimientos y competencias para el gestor de Marketing tanto para su comprensión como para administrar un equipo de profesiones que se adhieren al Marketing (como químicos, productores musicales, entre otros) que lo acompañe en el diseño e implementación de lo sensorial.

- En la planeación, el gestor de marketing sensorial debe incluir dentro de los objetivos tradicionalmente perseguidos, lograr el enganche emocional de los consumidores con la marca a través de los sentidos.

- En la gestión de marca, principalmente debe velar por la coherencia entre visión, principios y valores de marca y cada una de las manifestaciones comerciales de la misma. Esto se logra a través del desarrollo de experiencias sensoriales que añadan valor a la marca desde el punto de vista del consumidor.

- En función de las capacidades vigentes de la compañía especialmente en cuanto a cumplimiento de promesas tanto en producto como en servicio, el gestor de Marketing debe definir si genera una firma sensorial única desde lo olfativo, lo sonoro, lo táctil o lo gustativo (adicional al visual tradicional de la marca), para enriquecer la comunicación con su consumidor.

- Teniendo en cuenta que el Marketing sensorial busca representar una marca a través de los sentidos, los procesos de gestión deben tener en cuenta los estímulos externos que se usen deben ser los apropiados y sin exageraciones, guardando coherencia con el producto, para lograr la respuesta esperada del consumidor.

- Estas decisiones sobre estrategias sensoriales, deben ser analizadas y articuladas con definiciones ya existentes en la compañía (propuesta de valor, la identidad de marca y tipo de relación con sus clientes a largo plazo).

- El gerente de Marketing debe, de igual forma, verificar a diario el funcionamiento de la aplicación sensorial, puesto que es un esfuerzo sólo surte efecto a largo plazo si se vigila constantemente.

- En la administración de la comunicación desde lo sensorial con sus consumidores, el gerente de 
Marketing no puede olvidar que hoy en día los consumidores exigen sostener diálogos con la marca, y en este caso específico son de tipo emocional; también exigen sorpresa por parte de esta. Esto obliga al gerente de Marketing a contemplar la experticia de su agencia en percepción, sensación, sentimiento, emociones, fantasía e imaginación para inspirar y permitirle participar al consumidor.
- Como parte de la gestión de Marketing y de marca, los efectos que pueden generar las estrategias de Marketing sonoro y olfativo deben ser evaluados en las diferentes dimensiones (neurológica, psicológica, fisiológica). De lo contrario sus consecuencias pueden generar efectos contrarios a los esperados: aversión al producto, espacio o servicio que se quiere potencializar. 


\section{REFERENCIAS BIBLIOGRÁFICAS}

Aaker, D., (2002). Construir Marcas Poderosas. 2 Ed. Barcelona: Ediciones Gestión 2000.

Abril, C., Avello, M. y Manzano, R., (2011). Marketing visual: amor a primera vista. Harvard Deusto. Marketing \& Ventas. No. 103.

Alcaide Casado, J. C. y Merino, M. J., (2011). Comunicación experiencial y sensorial: algunos ejemplos de aplicación. Harvard Deusto. Business Review.

Álvarez del Blanco, R. (2011b). Fusión perfecta: neuromarketing. España: Prentice Hall.

Álvarez del Blanco, R., (2011a). Branding hoy: estrategias que funcionan. Marca multisensorial, espléndidamente lúcida. p26 a 33. Harvard Deusto. Marketing \& Ventas. No 106.

Aromarketing.com, (2011). El Marketing sensorial - A mayor vinculación con los sentidos, mayores ventas. Recuperado el 9 de 10 de 2011, disponible en: http://www.aromarketing.es/noticias/371-el-marketing-sensoriala-mayor-vinculacion-con-los-sentidos-mayores-ventas

Ascolfa/lcfes, (2010). Marco de referencia de los exámenes de Estado de Calidad de la Educación Superior en Administración (ECAES) (SABER PRO). Bogotá: Guía Publicidad y Asociados Ltda.

Avello, M., Gavilán, D. y Abril, C., (2011). Marketing auditivo: a qué suena una marca? Harvard Deusto. Marketing \& Ventas. No. 103.

Bonadeo, M., (2005). Odotipo: historia natural del olfato y su función en la identidad de marca. Colección investigación y tesis, Facultad de comunicación Universidad Autral - 1a ed. Buenos Aires: Editorial Universidad Austral.

Braidot, N., (2008). Neuromarketing: por qué tus clientes se acuestan con otro si dicen que les gustas tú? Barcelona, España: Ed. Gestión 2000.

Costa, J., (2010). La marca: creación, diseño y gestión. México: Trillas

Elder, R., Ayydinoglu, N., Barger, V., Caldara, C., Chun, H., Lee, C., y otros. (2010). A Sense of Things to ComeFuture Research Directions in Sensory Marketing.

Eysenck, H., (1992). Fundamentos biológicos de la personalidad. Barcelona: Fontanella. 
Gavilán, D., Abril, C. y Serra, T., (2011). Marketing olfatorio: el olor de los deseos. Harvard Deusto. Marketing \& Ventas. No. 103.

Gobé, M., (2001). Branding emocional. El nuevo paradigma para conectar las marcas emocionalmente con las personas. Barcelona: Divine Egg Publicaciones.

Hitt, M., Black, J. y Porter, L., (2006). Administración. 9 Ed. México: Pearson Education.

Hultén, B., Broweus, N. y Van Dijk, M.. (2009). Sensory Marketing. London: Palgrave Macmillan.

Keller, K., (2008). Administración estratégica de Marca. 3 Ed. Naucalpan de Juárez: Pearson Educación de México S.A.

Krishma, A., (2010). Sensory Marketing: research on the sensuality of products. New York USA: Taylor \& Francis Group.

Lindstrom, M., (2008). Brand Sense. New York: Simon \& Schuster.

Lindstrom, M., (2009). Compradicción: verdades y mentiras acerca de por qué las personas compran. (A. Arias, Trad.) Bogotá: Grupo Editorial Norma.

Manzano, R., Serra, T. Y Gavilán, D., (2011). Marketing sensorial: comunicar a través de los sentidos en marketing sensorial: nuevas claves para su gestión. Harvard Deusto. Marketing y Ventas. No. 103.

Mejía, J., (2011a). El Bon Bon que es Boom. Borradores de Administración. Bogotá: CESA.

Mejía, J., (2011b). Tiempo de Chocorramo. Manuscrito presentado para su publicación.

Mitchell, D., Kahn, B.E., Knazko, S.C., (1995). There's something in the Air: Effects of Congruent or Incongruent Ambient odor on Consumer Decision Making, Journal of Consumer Research, Vol. 22, p 229-238.

Mulligan, E. N., (2010, 21 de Junio). Why Your Brand Should Have a Purpose. Ad and Marketing News. AdvertisingAge. Recuperado el 1 de agosto de 2010 Disponible en: http://adage.com/columns/article?article_ id $=144535$

Ospina, J. y Samper, F., (2010). El efecto de los aromas en la recordación de marca. Mejores proyectos de grado, Facultad de Administración, Universidad de los Andes. No.49. Bogotá, D.C.: Guías de Impresión.

Peris, A. y Cuadrado, D., (2008). Los 5 sentidos de la venta- un camino sensorial para vender. Bogotá: Grupo Editorial Norma.

Pradeep, A.K., (2010). The Buying Brain: secrets for selling to the subconscious mind. New Jersey, USA: .John Wiley \& Sons, Inc., Hoboke 
Resa, S., (2011, 17 de febrero). La compra de los sentidos. Factores determinantes, estrategias, tendencias y expectativas. Recuperado el 11 de septiembre de 2011. Disponible en: http://www.mercasa.es/noticias/post/ la_compra_de_los_sentidos.

Ruiz, J., (director y coautor), (s.f.). The customer experience: una visión multidimensional del marketing de experiencias. Libro Colaborativo: \#CEMbook, eBook. España.

Salcedo, A., (2009). Con los cinco sentidos. Seducción e instinto de compra: razones para consumir. Recuperado el 11 de 09 de 2011. Disponible en: http://www.inpsicon.com/lo-que-hacemos/revista-el-consumidor/ articulos/634-con-los-cinco-sentidos-seduccion-e-instinto-de-compra-razones-para-consumir.html

Sarafoleanu, C., Mella, C., Georgescu, M., y Perederco, C., (2009). The importance of the olfactory sense in the human behavior and evolution. Journal of Medicine and Life 15; 2(2): 196-198.

Sebriano, E., (2012, 10 de junio). Marketing sensorial y emocional: multiplicar emociones relacionadas con las marcas por medio de la creación de experiencias seonsoriales en cada uno de los sentidos. Eduardo Sebriano: Marketing Sensorial, Innovación, Consumidores y Estrategias. Recuperado el 10 de junio de 2012. Disponible en: http://www.sebriano.blogspot.com/2012/06/marketing-sensorial-y-emocional.html

Serra, T., Manzano, R., Avello, M., (2011). Tacto y gusto: generar sensaciones a través del contacto directo con el producto. Harvard Deusto. Marketing y Ventas. No. 103.

Schmitt, B., (2006). Experiential Marketing, Cómo conseguir que los clientes identifiquen en su marca. Ediciones Deusto.

Smilansky, S., (2009). Experiential Marketing: A practical guide to interactive brand experiences. London and Philadelphia: Kogan Page

Spangenberg, E., Crowly, A., y Henderson, P., (1996). Improving the Store Environment: Do Olfactory Cues Affect Evaluations and Behaviors? The Journal of Market , 60 (2), p 67-80.

Underhill, P., (2007). El placer de comprar, 24 horas en un centro comercial. Gestión 2000.

vidaextra.com, (2009). Jugando con la parada del autobús. Blog de noticias sobre videojuegos y consolas. Recuperado el 1 de septiem,bre de 2011 desde: http://www.vidaextra.com/videos/jugando-con-la-parada-delbus)

Villar, C., (2009). La mente del consumidor desde una mirada psicoanalítica. Experiencia del Consumidor. Recuperado el 04 de Mayo de 2010. Disponible en: http://cem.bligoo.com/content/view/441556/La-mente-delconsumidor-desde-una-mirada-psicoanalitica.html\#content-top. 